\title{
Aumentando a eficiência das colunas de HPLC por meio da diminuição do diâmetro das partículas da fase estacionária: até onde?
}

\author{
Fernando M. Lanças \\ Instituto de Química de São Carlos, Universidade de São Paulo \\ Cep 13560-970, São Carlos, SP, Brasil \\ e-mail: flancas@iqsc.usp.br
}

Resumo

Após o desenvolvimento da instrumentação para a cromatografia líquida moderna (HPLC ou CLAE), durante as duas primeiras décadas, muito pouco foi feito para ampliar as possibilidades analíticas da técnica. A partir da terceira década, um esforço maior foi feito no desenvolvimento de partículas menores, mais uniformes e de formato mais reprodutível, objetivando um aumento na eficiência das colunas e, como consequência, da resolução cromatográfica. Entretanto, a diminuição drástica do tamanho das partículas - de $10 \mu \mathrm{m}$, na década de 1970, para 1,7 $\mu \mathrm{m}$, na presente década - ocasionou um aumento considerável na pressão do sistema, usualmente atingindo valores superiores aos suportados pelos instrumentos convencionais. No presente trabalho, questiona-se até que tamanho as partículas da fase estacionária poderão ser reduzidas, assim como se essa é a única forma de aumentar a resolução cromatográfica.

Palavras-chave

HPLC; U-HPLC; micropartículas; eficiência das colunas.

\section{Increasing the efficiency of HPLC columns by decreasing the particles diameter: till where?}

\section{Abstract}

Since the development of instrumental modern liquid chromatography (HPLC), very few was done during the first two decades in order to extend the possibilities of the technique. During the third decade an effort was done aiming the development of smaller, more uniform, and more reproducible particles to increase the efficiency of the columns and, as a consequence, the chromatographic resolution. However, the dramatic reduction in the particles size - from 10 to $1.7 \mu \mathrm{m}$ - produced a considerable increase in the column backpressure, achieving values above those supported by conventional HPLC instruments In the present work we discuss the lowest limit size to which the particles should decrease, as well as if this is the best way to increase the chromatographic resolution.

Keywords

HPLC; U-HPLC; microparticles; column efficiency. 


\section{Introdução}

A eficiência de uma coluna cromatográfica é usualmente medida pelo número de pratos $(\mathrm{N})$, o qual é determinado diretamente do cromatograma por meio da relação entre o tempo de retenção de um composto e a largura de sua base $^{1}$ :

$$
N=\left(\frac{t_{R}}{w_{b}}\right)^{2} \times 16
$$

em que $\mathrm{N}$ é o número de pratos da coluna, uma medida da eficiência; $t_{R}$ é o tempo de retenção do analito empregado na medida e $\mathrm{w}_{\mathrm{b}}$, a largura na base desse mesmo analito.

Apesar da simplicidade inequívoca deste enfoque, existem muitas críticas a ele, a começar pelo fato de que o número de pratos (eficiência) dependerá do composto escolhido e das condições analíticas, uma vez que o tempo de retenção e a largura variam para cada composto e também com as condições experimentais. Tal problema pode ser facilmente evitado estabelecendo-se o composto a ser empregado para o cálculo de $\mathrm{N}$ e as condições cromatográficas. Ainda, assim, surgem outras dificuldades como o fato de que muitas variáveis influenciam a eficiência de uma coluna, como o seu comprimento (L). Comparando-se duas colunas com mesma fase estacionária, aumentando-se o comprimento aumenta-se o número de pratos $(\mathrm{N})$, uma vez que aumentará o número de equilíbrio do analito entre as fases estacionária e móvel ${ }^{1}$.

Outro parâmetro que exerce bastante influência na eficiência de uma coluna é o tamanho (diâmetro) e a distribuição das partículas da fase estacionária. No início da HPLC, as partículas empregadas como fase estacionária eram sintetizadas principalmente a partir de sílica gel, de diâmetro típico em torno de $10 \mu \mathrm{m}$ (início da década de 70), com grande tolerância para a faixa aceita (geralmente entre cerca de 7 e $15 \mu \mathrm{m}$ ). Com a intenção de melhorar a eficiência das colunas, o tamanho típico das partículas foi reduzido para $7 \mu \mathrm{m}$; na última década do século passado, o tamanho médio nominal (uma vez que na prática existe uma faixa) das partículas já era de $5 \mu \mathrm{m}$.

Desde o surgimento da teoria dos pratos para cromatografia ${ }^{2}$, originalmente desenvolvida para cromatografia gasosa e depois adaptada para HPLC, ficou bastante óbvio que a diminuição do tamanho das partículas seria o caminho natural para o aumento do número de pratos. Em sua versão original, van Deemter normalizou o cálculo da eficiência de uma coluna dividindo seu comprimento $(\mathrm{L})$ pelo o número de pratos $(\mathrm{N})$ resultado denominando de altura equivalente a um prato $(\mathrm{H})$, ou seja,

$$
H=\frac{L}{N}
$$

Essa proposta permitia então comparar colunas de diferentes comprimentos (L) e tamanhos de partículas (dp), uma vez que, independente dos detalhes, quanto menor o valor de $\mathrm{H}$, maior será $\mathrm{N}$ e, portanto, a eficiência da coluna. $\mathrm{Na}$ forma original, van Deemter desenvolveu uma equação empírica em que associou o valor de $\mathrm{H}$ com vários parâmetros, agrupados na forma de constantes $\mathrm{A}, \mathrm{B}$ e C.

$$
H=A+\frac{B}{\mu}+C \mu
$$

Demonstrou também a dependência de $\mathrm{N}$ com a velocidade linear média da fase móvel $(\mu)$, ou seja,

$$
\mu=\frac{L}{t_{0}}
$$

em que $\mu$ é a velocidade linear média da fase móvel; L, o comprimento da coluna e to, o tempo morto da coluna (tempo para eluir um composto pouco retido). 
O termo A depende de dois fatores principais: do quadrado do tamanho médio das partículas (dp) e do fator de empacotamento da coluna (f). Assim, $\mathrm{H}$ depende diretamente do tamanho das partículas, ou seja,

$$
H \alpha d p^{2}
$$

Aumentando-se o tamanho das partículas, aumenta-se $\mathrm{H}$ e, portanto, diminui-se a eficiência de N. Dessa forma, e em consonância com a teoria, a maneira ideal de aumentar a eficiência da coluna seria a diminuição do tamanho das partículas.

\section{O efeito da diminuição do tamanho das partículas na eficiência}

Uma vez obtida uma eficiência adequada com as partículas de $5 \mu \mathrm{m}$, (por volta de 1985), pouca inovação apareceu durante cerca de uma década na área, a não ser pelo preparo de partículas de sílica com diâmetro entre 3,0 e 3,5 $\mu \mathrm{m}$ (por volta de 1992), utilizando praticamente a mesma tecnologia da década anterior. Essa diminuição no tamanho das partículas possibilitou uma melhora nas separações mais complexas, com diminuição do tempo de análise, sem necessidade de modificações instrumentais consideráveis, ou seja, a maioria dos cromatógrafos fabricados na última década do século passado eram adequados para operação com essas colunas. Entretanto, na prática, esse era o menor tamanho de partícula que poderia ser utilizada como fase estacionária, sem necessidade de mudanças significativas nos equipamentos convencionais de HPLC. A principal limitação para a diminuição do tamanho das partículas, e consequente aumento de $\mathrm{N}$, é o bem conhecido fato de que a diminuição no tamanho das partículas provoca um aumento significativo na pressão do sistema, pois

$$
\Delta P=\frac{\phi L \eta}{100} \frac{1}{d p^{2}}
$$

em que: $\Delta \mathrm{P}=$ variação da pressão na coluna (diferença entre a pressão de entrada e de saída); $\eta=$ viscosidade da fase móvel; e dp = diâmetro médio das partículas.

Nesse caso, a queda de pressão $(\Delta \mathrm{P})$ varia inversamente com o tamanho das partículas (dp): a diminuição no dp ocasiona aumento na pressão do sistema.

As pressões geradas por partículas de diâmetro inferior a $3 \mu \mathrm{m}$ eram usualmente superiores àquelas suportadas pela maioria dos cromatógrafos comercializados no início deste século. A adequação das bombas, injetores, celas de detecção, sistemas de dados e outros detalhes instrumentais permitiu o uso de colunas de diâmetro menor que $3 \mu \mathrm{m}$ na nova geração de cromatógrafos. Para salientar essa mudança de filosofia de trabalho, com o uso de colunas de menor comprimento (tipicamente $\leq 5 \mathrm{~cm}$ ) e partículas de diâmetro menor (usalmente 1,7 ou $1,8 \mu \mathrm{m}$ ), cunhou-se a sigla U-HPLC (Ultra- High Performance Liquid Chromatography), com a intenção de designar o novo sistema, o qual pode operar com partículas de menor tamanho, uma vez que suporta pressões mais elevadas que a HPLC convencional. A maior parte dos fabricantes de equipamentos aderiu rapidamente à nova modalidade de HPLC, comercializando equipamentos com diferentes siglas.

Além do aumento da eficiência das colunas (N) por meio da redução de $\mathrm{H}$, a diminuição do tamanho das partículas traz um benefício adicional: permite operar-se com fases móveis a elevada velocidade linear média (ou fluxo/vazão), sem perda significativa de eficiência. Assim, 
torna-se mais próximo o sonho dos cromatografistas, o qual seria trabalhar com o máximo de eficiência e menor tempo de análise possível.

A Figura 1 ilustra a variação de $\mathrm{H}$ em função do tamanho das partículas para colunas que contêm partículas de dp $=5 \mu \mathrm{m}, 3,5 \mu \mathrm{m}$ (HPLC) e $1,8 \mu \mathrm{m}$ (U-HPLC).

Observa-se uma notável diminuição no valor de $\mathrm{H}$ para as partículas de 1,8 $\mu \mathrm{m}$ (U-HPLC) em relação às de 3,5 e $5 \mu \mathrm{m}$ empregadas no modo HPLC convencional. Adicionalmente, nota-se que o aumento da velocidade linear média (ou fluxo volumétrico) praticamente não exerce influência no valor de $\mathrm{H}$ para partículas de $1,8 \mu \mathrm{m}$ (U-HPLC), enquanto aumenta para as partículas de 3,5 $\mu \mathrm{m}$ e, de forma mais acentuada, para as de $5 \mu \mathrm{m}$, as quais operam no modo HPLC convencional.

\section{O efeito da diminuição do tamanho das partículas no tempo de análise}

Conforme mostra a Figura 1, diminuindo-se o tamanho das partículas, pode-se trabalhar com fluxos volumétricos maiores, sem perda de eficiência, diminuindo o tempo de análise. Isso pode ser melhor visualizado pela equação que relaciona o tempo de retenção de um composto com o tamanho das partículas da fase estacionária ${ }^{3}$.

$$
t_{R}=\frac{(1+k) N h}{D m \mu} \cdot d p^{2}
$$

em que: $t_{R}=$ tempo de retenção; $k=$ fator de retenção; $\mathrm{N}=$ número de pratos; $\mathrm{h}=$ altura de $\mathrm{um}$ prato; $\mathrm{Dm}=$ difusão do analito na fase móvel; $\mathrm{dp}=$ diâmetro médio das partículas da fase estacionária.

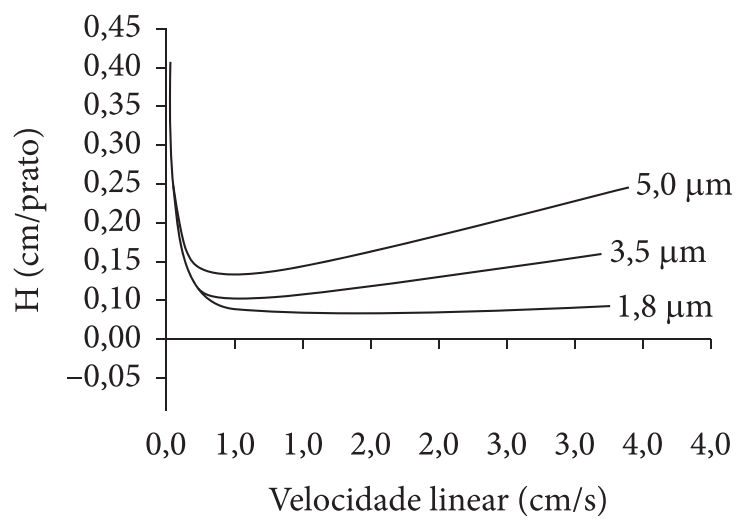

Figura 1 Curvas de van Deemter para colunas que contêm partículas de diferentes valores de $d p$.

Uma vez que o valor do tamanho da partícula da fase estacionária é diretamente proporcional e quadrático na fórmula, uma pequena diminuição no dp ocasionará acentuada diminuição no tempo de retenção do analito (e, portanto, no tempo total de análise).

\section{Quo Vadis?}

Apesar de a diminuição do tamanho das partículas em HPLC conduzir a uma maior eficiência das colunas, o preço a pagar é a elevação da pressão no sistema, requerendo uma instrumentação especial. Os sistemas mais antigos, dimensionados para trabalhar com pressões de até cerca de 6.000 psi (400 bar), acomodam, sem problemas, colunas que contêm partículas com diâmetro de até $5 \mu \mathrm{m}$ e colunas curtas (até $5 \mathrm{~cm}$ de comprimento) que contêm partículas de 3 a 3,5 $\mu \mathrm{m}$. Partículas menores que essas tendem a aumentar muito a pressão no sistema e provocar rápido desgaste do instrumento, requerendo o uso de equipamentos desenvolvidos para U-HPLC.

Tecnologias mais recentes de preparo de partículas para HPLC, desenvolvidas a partir de 2003, especialmente aquelas baseadas no emprego de sol-gel, têm permitido a síntese de 
partículas de menor tamanho com menor faixa de dispersão, ou seja, praticamente todas as partículas contendo a mesma forma e tamanho ${ }^{4}$. Essas novas possibilidades melhoram muito o desempenho das colunas, permitindo um aumento dramático na eficiência, mesmo empregando colunas mais curtas - significando análises mais rápidas.

A Figura 2 ilustra uma separação cromatográfica obtida em coluna que contêm, como fase estacionária, sílica com diâmetro igual a $1 \mu \mathrm{m}^{5}$.

Enquanto em HPLC convencional, uma coluna tipicamente gera em torno de 20.000 a 30.000 pratos, a coluna da Figura 2 gera em torno de 500.000 pratos de eficiência, dependendo do composto escolhido para o cálculo (Tabela 1). Obviamente, trata-se de uma escala de eficiência extremamente elevada, mesmo comparando com a HPLC convencional, que é referida como Cromatografia Líquida de Alta Eficiência (CLAE).

A Figura 3 ilustra a fotomicrografia de partículas de sílica de $1 \mu \mathrm{m}$ sintetizadas pela tecnologia da sílica gel ${ }^{6}$, em que se observa a excelente esfericidade e uniformidade do tamanho das partículas.

A partir desses dados, torna-se inevitável a pergunta: para onde vamos (Quo Vadis)?, ou seja, até que tamanho poderemos diminuir as partículas e utilizarmos equipamentos comercialmente disponíveis para obtermos uma melhora na separação de misturas complexas (maior $\mathrm{N}$ ) ou análises mais rápidas (menor $\mathrm{t}_{\mathrm{R}}$ ) ? Uma vez que os primeiros resultados obtidos com partículas de tamanho igual ou inferior a $1 \mu \mathrm{m}$ são muito recentes e foram conseguidos em equipamentos montados no laboratório, ainda é cedo para se estabelecer um limite para a U-HPLC. Porém, sem dúvida, a rapidez com que essa área de pesquisa caminha, tudo sugere que, em breve - provavelmente na década que se inicia - um novo salto, similar ao que ocorreu com a U-HPLC, deverá ocorrer.
Tabela 1 Número de pratos gerados pela coluna apresentada na Figura 2.

\begin{tabular}{cc}
$\begin{array}{c}\text { Número do composto } \\
\text { no cromatograma }\end{array}$ & $\begin{array}{c}\text { Número de pratos } \\
\text { (determinado) }\end{array}$ \\
\hline 1 & 600.000 \\
2 & 550.000 \\
3 & 400.000 \\
4 & 400.000 \\
5 & 480.000 \\
\hline
\end{tabular}

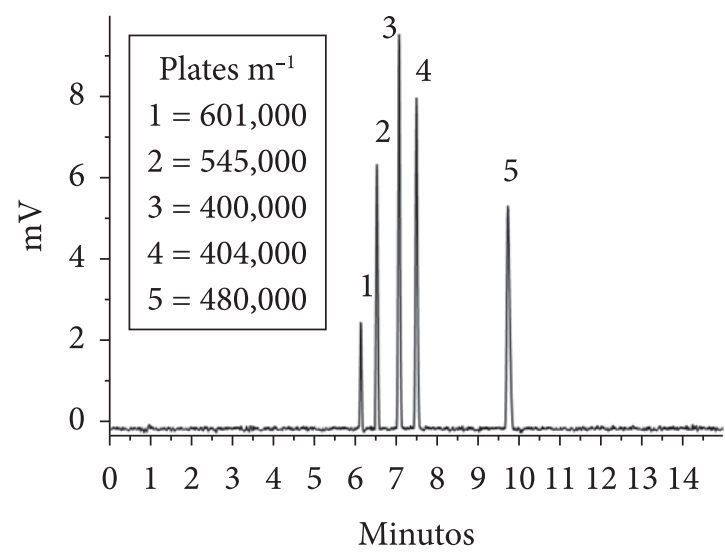

Figura 2 Análise de uma mistura de padrões analíticos empregando uma coluna empacotada com partículas de sílica de $1 \mu \mathrm{m}(\mathrm{L}=30 \mathrm{~cm}$; di=30 $\mu \mathrm{m})$. Fase móvel Água (0.1 \% TFA)/acetonitrilo (90:10 v.v $\left.\mathrm{v}^{-1}\right)$; detector UV detection, $215 \mathrm{~nm}$; Pressão 15.000 psi. Analitos: 1 = Ácido ascórbico; 2 = Hidroquinona; 3 = Resorcinol; 4 = Catecol; e $5=4$-metil catecol. (modificado da ref. 5).

\section{Questões para reflexão}

Não há dúvida de que a diminuição no tamanho das partículas ocasiona uma melhora na eficiência do sistema. Isso é previsto pela teoria da cromatografia e amplamente comprovado na prática. A pergunta que merece reflexão é: até que ponto vale a pena pagar para diminuir acentuadamente o tamanho das partículas? As seguintes questões são deixadas para reflexão dos leitores interessados em melhorar o desempenho de suas análises, mas não necessariamente a qualquer custo. 


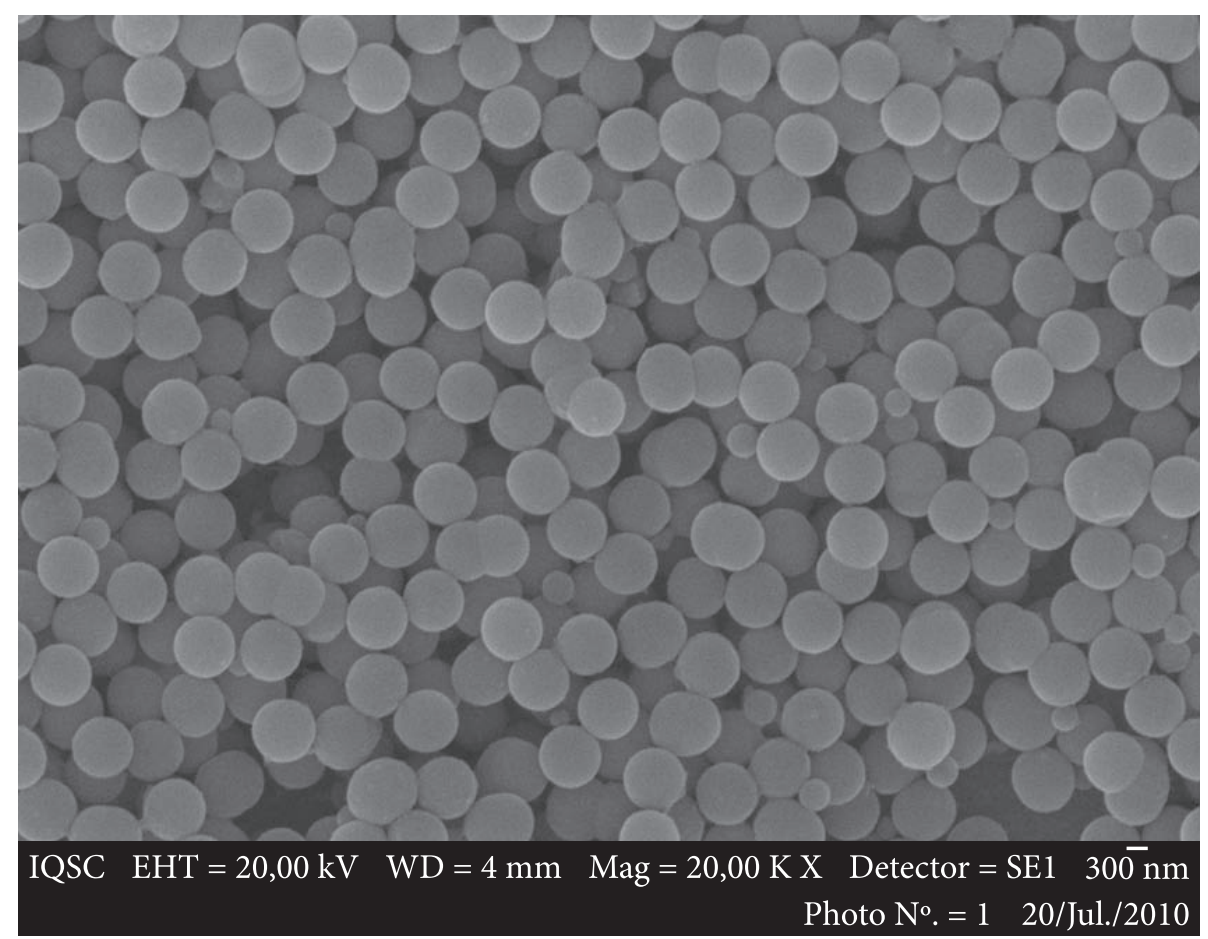

Figura 3 Fotomicrografia de partículas de sílica de diâmetro nominal $1 \mu \mathrm{m}$, sintetizadas por meio da tecnologia sol-gel ${ }^{6}$.

\subsection{Você efetivamente necessita aumentar} a eficiência da coluna a ponto de aposentar um cromatógrafo HPLC convencional que está operando bem com partículas de $3 \mu \mathrm{m}$ e colocar, em seu lugar, outro operando com partículas de 1,8 $\mu \mathrm{m}$ ?

Somente você pode responder essa questão. Antes disso, lembre-se de que não é apenas a eficiência da coluna que conta em uma separação, já que a resolução depende de outros fatores igualmente importantes. A equação da resolução, Rs, estabelece que uma separação, para ter sucesso, depende de 3 fatores, sendo $\mathrm{N}$ apenas um deles ${ }^{1}$ :

$$
R_{S}=1 / 4 \sqrt{N} \times \frac{\alpha-1}{\alpha} \times \frac{k}{1+k}
$$

\section{Eficiência Seletividade Retenção}

Ainda assim, a resolução (Rs) depende da raiz quadrada de $\mathrm{N}$ e não diretamente desse fator. Dependendo da separação, é mais interessante modificar o termo $\alpha$ (usualmente mudando-se a composição química da fase estacionária) ou k (mudando-se a composição da fase móvel) e continuar usando, com sucesso, o mesmo equipamento, até que efetivamente uma mudança radical de estratégia analítica seja necessária.

\subsection{O uso de partículas de diâmetro menor restringe o comprimento das colunas (colunas com partículas menores que $2 \mu \mathrm{m}$ tipicamente não possuem comprimento superior a $5 \mathrm{~cm}$ )}

Caso o tempo de análise não seja sua maior preocupação, mas sim a melhor separação dos componentes, colunas mais longas do que $5 \mathrm{~cm}$ poderão ser necessárias. Nesse caso, partículas com dp igual a $3 \mu \mathrm{m}$ poderão ser mais interessantes por permitirem aumentar o comprimento da coluna, aumentando-se significativamente $\mathrm{N}$ e $\mathrm{R}$, sem que o limite de pressão do equipamento seja atingido. 


\subsection{O uso de colunas contendo partículas superficialmente porosas (core shell, porous shell, ou SPC) permitem o uso de colunas com diâmetro de partículas menores, porém com menor queda de pressão do que as partículas não porosas}

Horvath e Lipsky, nos anos $1960^{[7]}$, recobriram esferas não porosas (shell cores), tais como pequenas contas (esferas) de vidro, com uma película de material sólido poroso. Nesse tipo de material, as moléculas da amostra migram através dessa camada relativamente fina, resultando em picos mais estreitos do que aqueles obtidos quando se empregam partículas totalmente porosas $^{8}$. Esse tipo de material foi denominado de pelicular (devido à película colocada na superfície) ou superficialmente porosas (devido às características da película).

Apesar de algumas tentativas para preparação de partículas superficialmente porosas ou peliculares com tamanho menor, a primeira demonstração prática de tal possibilidade ocorreu somente no início desta década ${ }^{9}$. Partículas de 5,0 $\mu \mathrm{m}$ de diâmetro total - incluindo uma camada porosa de $0,25 \mu \mathrm{m}$ de espessura - contendo poros de $30,0 \mathrm{~nm}$ foram produzidas e empregadas na separação de várias moléculas, aproveitando as pequenas distâncias de difusão de solutos de baixa difusividade. Mais recentemente, Kirkland ${ }^{10}$ desenvolveu partículas de C-8 e C-18 com diâmetro total de 2,7 $\mu \mathrm{m}$, com uma camada porosa de $0,5 \mu \mathrm{m}$ de espessura, contendo poros de $90 \AA$. . A queda de pressão $(\Delta \mathrm{P})$ típica dessas colunas é bastante inferior àquelas atingidas por partículas não porosas de $1,7-1,8 \mu \mathrm{m}$ empregadas em U-HPLC, enquanto a cinética da transferência de massa (termo $\mathrm{C}$ da equação de van Deemter) ${ }^{8}$ na fina camada porosa da superfície dessas partículas é bastante favorecida, permitindo análises rápidas.
Concluindo, existem, no momento, vários enfoques para se obter maior sucesso em uma análise cromatográfica por meio do aumento da eficiência da coluna $(\mathrm{N})$. Entretanto, essa não é a única forma de melhorar a separação em cromatografia e, frente às várias opções disponíveis, o analista precisa refletir a respeito de qual delas é a mais apropriada para seu caso, pois, nem sempre, o que está na moda é a melhor solução.

\section{Referências}

1 Lanças FM. Cromatografia líquida de alta eficiência. Ed. Átomo; 2009.

2 Van Deemter JJ, Zviderweg F, Klinkenberg A. Longitudinal diffusion and resistance to massa transfer as causes of non ideality in chromatography. Chemical Engineering Science 1956; 5:271.

3 Lanças FM. Estratégias para diminuição do tempo de análise em Cromatografia Líquida Moderna. Scientia Chromatographica 2009; 1(4):39.

4 Lanças FM. Cromatografia líquida com interação hicrofílica (HILIC). Scientia Chromatographica 2010; 2(1):49.

5 Xiang Y. Capillary liquid chromatography usindo micro size particles [Ph.D. dissertation]. Local: Brigham Young University; 2004.

6 Nazario C, Lancas FM. Resultados não publicados da tese de Doutorado de C. Nazario, sob orientação de F.M. Lancas no Instituto de Química de São Carlos, USP; 2010.

7 Horvath CS, Lipsky SR. Use of ion-exchange chromatography for the separation of organic compounds. Nature (London) 1966; 322:748-749.

8 Lanças FM. O Renascimento das particulas superficialmente porosas ("core shell particles") em HPLC. Scientia Chromatographica 2010; 2(2)47.

9 Kirkland JJ, Truszkowski FA, Dilks CH, Engel GS. Superficially porous silica mircospheres for fast highperformance liquid chromatography of macromolecules. Journal of Chromatography A 2000; 890:3-13.

10 Kirkland J, Langlois TJ, DeStefano JJ. Fused core particles for HPLC columns. American Laboratory 2007; 39:18-21. 